> Les micro-ARN représentent une découverte majeure dans le domaine de la régulation de l'expression des gènes. Ils participent à la régulation de l'expression de protéines en inhibant la traduction et/ou en induisant la dégradation de leurs ARNm correspondants. L'étude de leurs fonctions a révélé leur rôle dans la prolifération cellulaire et le développement chez les métazoaires. La recherche des altérations moléculaires en cause dans l'apparition de cancers a permis d'identifier ces molécules en tant que nouveaux acteurs de la transformation néoplasique. Ainsi, des micro-ARN présentant les caractéristiques d'oncogènes (miR-17-92) ou celles de suppresseur de tumeurs (let-7) ont été découverts ces dernières années. <

\section{Micro-ARN : oncogènes et suppresseurs de tumeurs}

Anne-Laure Finoux, Pascal Chartrand

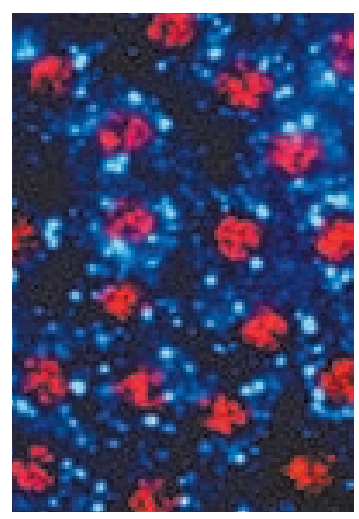

Département de Biochimie, Université de Montréal, 2900, Édouard-Montpetit, Montréal, Québec, H3C 3J7 Canada. p.chartrand@umontreal.ca
Les micro-ARN (miARN) sont de petits ARN non codants identifiés récemment comme des éléments clés de la régulation de l'expression des gènes. Depuis leur découverte chez le nématode Caenorhabditis elegans en 1993 [1], des études ont révélé, chez l'humain, la présence de plus de 500 miARN pouvant cibler potentiellement près de $30 \%$ des ARNm humains [2]. Chez les plantes et les métazoaires [3], les miARN constituent une classe abondante de petits ARN de 18 à 24 nucléotides de longueur. Un miARN lie une ou plusieurs séquences précises dans la région 3'-UTR d'un ARNm cible et recrute ainsi le complexe RISC (RNA-induced silencing complex) vers cet ARNm (Figure 1). Il est admis que si la complémentarité entre le miARN et sa cible est parfaite, la protéine Ago2 - un composant du complexe RISC - clive l'ARNm, qui est alors dégradé par les enzymes des voies de dégradation. En revanche, si la complémentarité est imparfaite, une répression de la traduction de I'ARNm cible est généralement observée. II existe cependant plusieurs cas pour lesquels une complémentarité imparfaite provoque une dégradation de l'ARNm cible, démontrant ainsi que cette règle n'est pas toujours respectée [4]. Une propriété supplémentaire des miARN est venue s'ajouter récemment à leur champ d'action. Deux études ont en effet proposé que certains miARN sont également capables d'activer spécifiquement la traduction de leurs ARNm cibles $[5,6]$.

La découverte de ces petits $A R N$ régulateurs a révolutionné l'étude des mécanismes biologiques liés au développement des organismes et aux maladies, notamment le cancer. En effet, jusqu'alors, les études se focalisaient essentiellement sur les gènes codant pour des protéines considérées comme étant les effecteurs uniques de la transformation cellulaire néoplasique. Depuis, de nombreuses études ont mis en évidence une corrélation entre la dérégulation de l'expression de certains miARN et certains cancers, pointant ainsi vers un nouveau type de molécule effectrice dans l'apparition et la progression des tumeurs.

\section{Les micro-ARN et leur rôle dans les cancers}

Les miARN participent à des étapes clés du développement des métazoaires; cependant, leur dérégulation va de pair avec l'apparition et le développement de certains cancers. Ainsi, des études consacrées à mesurer l'expression des miARN ont révélé des altérations dans des échantillons cliniques de patients atteints de cancers [7]. La cartographie des gènes codant pour ces miARN a confirmé ces observations, en révélant qu'ils sont présents dans des régions chromosomiques amplifiées ou délétées dans les cellules tumorales [8]. Leur existence a également permis de comprendre pourquoi certaines de ces régions génomiques n'étaient 


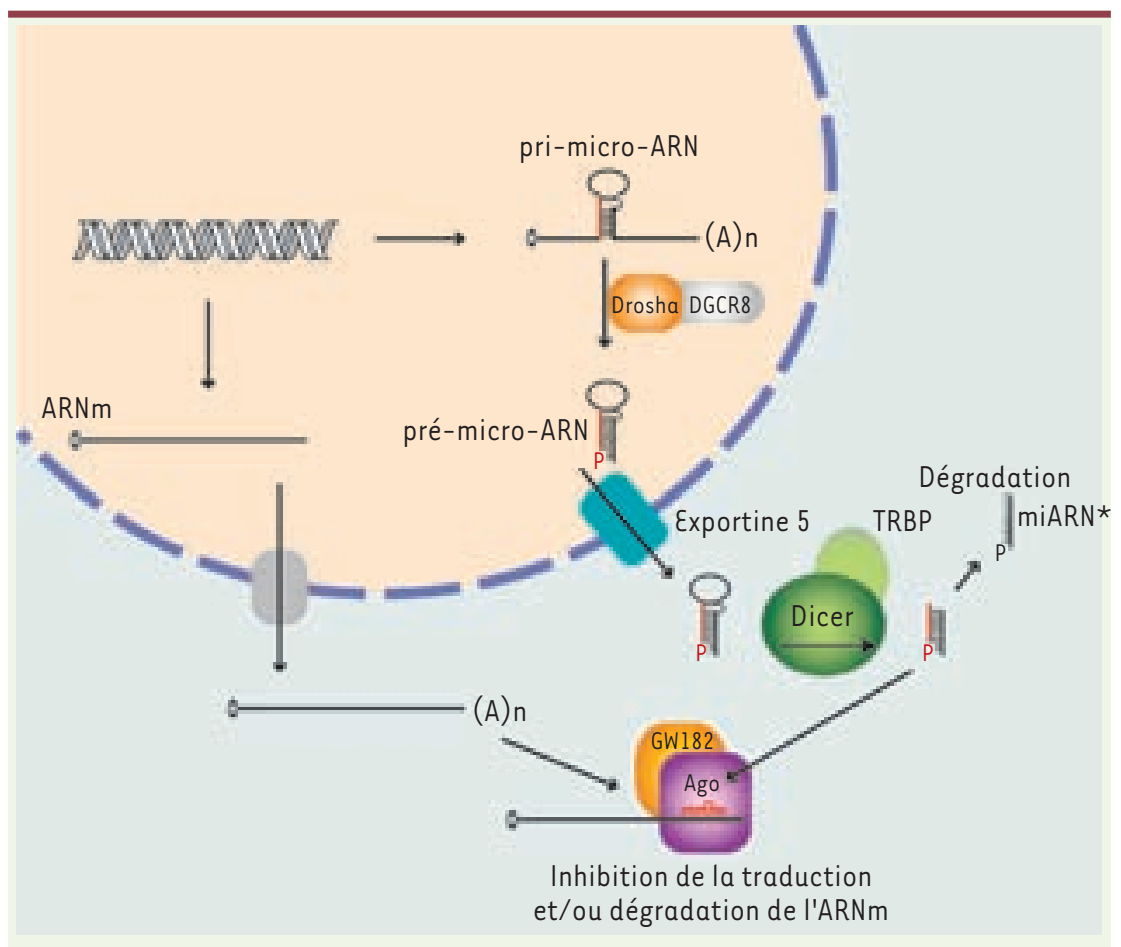

Figure 1. Biogenèse des micro-ARN. Les miARN sont synthétisés presque exclusivement par I'ARN polymérase II sous la forme de longs précurseurs appelés pri-micro-ARN. Ils possèdent leur propre unité de transcription ou sont produits à partir de régions introniques d'ARNm. Certains miARN sont synthétisés à partir d'un même pri-micro-ARN et sont sous la dépendance d'un même promoteur, on parle alors de miARN polycistronique. Le primiARN est modifié par l'ajout d'une coiffe en 5 ' et d'une queue poly (A) en 3', tout comme les ARNm, mais n'est pas traduit en protéine. II se replie et adopte des structures secondaires en tige boucle, puis subit une étape de maturation par un complexe formé d'une RNase de type III, Drosha, et de son cofacteur DGCR8. II en résulte alors une molécule de 60 à 70 nucléotides possédant comme caractéristiques une extrémité 5' phosphate et une extrémité 3' présentant deux nucléotides sortants. Cette molécule intermédiaire, appelée pré-micro-ARN (pré-miARN), est ensuite exportée du noyau vers le cytoplasme par un transporteur de la famille Ran, l'exportine-5. Une dernière étape de maturation est nécessaire afin de produire le miARN. Dicer, une seconde RNase de type III, est responsable du clivage de la boucle terminale du pré-miARN. Un duplex double brin constitué du miARN et de son complémentaire appelé miARN* est alors produit. Le miARN mature est incorporé dans le complexe RISC (Ago/GW182) tandis que son complémentaire est dégradé.

associées ni à un suppresseur de tumeurs ni à un oncogène connu. La recherche des fonctions biologiques de ces miARN et leurs rôles dans la tumorigenèse ouvre un nouveau champ d'investigation pour la recherche biomédicale en raison du lien établi entre l'expression de miARN et certains cancers. Bien que plusieurs miARN associés à des cancers aient été décrits ces dernières années, nous ne discuterons dans cet article que de deux exemples, miR-17-92 et let-7, qui comptent parmi les mieux caractérisés et qui illustrent la façon dont les petits ARN peuvent agir comme oncogènes ou suppresseurs de tumeurs.

\section{miR-17-92 : un polycistron de miARN oncogène}

Les nouvelles méthodes d'analyse d'expression des gènes par micropuces ont établi que les profils des miARN pouvaient être utilisés comme marqueurs moléculaires de tumeurs puisque ceux-ci varient entre tissus sains et cancéreux. Les miARN du polycistron miR-17-92 sont les premiers à avoir été identifiés par cette technique comme étant potentiellement oncogéniques [9]. Le locus miR-17-92 code pour six miARN (miR-17-5, miR-18a, miR-19a, miR-20a, miR-19b, et miR-92) exprimés à partir d'une même unité de transcription (d'où le nom de polycistron) située dans le troisième intron d'une phase ouverte de lecture (C13orf25). II existe dans le génome humain deux autres polycistrons de miARN, miR-106a-363 et miR-106b-25, qui codent pour des miARN homologues à ceux de miR-17-92 [10] et qui auraient également des propriétés oncogéniques (voir revue de J.T. Mendell [11] pour plus de détails).
Ces miARN participent au développement de la souris puisqu'une délétion du locus miR-17-92 rend la souris non viable, possiblement à cause d'une hypoplasie des poumons et d'une anomalie cardiaque [12]. Le polycistron miR-17-92 semble aussi être important pour le développement des cellules lymphoïdes puisque sa délétion dans les cellules $B$ progénitrices mène à une diminution de la survie des lymphocytes B [12] et que sa surexpression dans les lymphocytes $B$ favorise plutôt leur prolifération [13]. Cette observation est en accord avec un rôle pro-prolifératif du polycistron, qui présente différentes propriétés d'un oncogène. En effet, d'une part, il est localisé dans une région fréquemment amplifiée dans plusieurs types de lymphomes et de cancers du poumon, la région 13q31-32 du chromosome $13[14,15]$ et, d'autre part, ces miARN sont surexprimés dans plusieurs cancers (Tableau I). Cette hypothèse a été confirmée dans un premier temps grâce à une souris modèle pour la formation de lymphomes (souris $\varepsilon \mu-M y c)$ puisque dans cet organisme, la surexpression simultanée de c-Myc et d'une forme tronquée du polycistron miR-17-92 accélère de façon dramatique l'apparition de lymphomes [9]. De même, la surexpression de ce polycistron dans les cellules progénitrices épithéliales du poumon favorise leur prolifération [16]. 


\begin{tabular}{|c|c|c|c|}
\hline Micro-ARN & Fonctions & Types de cancer* & ARNm cibles* \\
\hline \multirow{2}{*}{$\begin{array}{l}\text { Polycistron } \\
\text { miR-17-92 }\end{array}$} & Oncogène & $\begin{array}{l}\text { Lymphomes des cellules B [15], cancer des poumons [14], } \\
\text { cancer de la prostate [39], cancer du côlon [39] }\end{array}$ & $\begin{array}{l}\text { ع2F1, PTEN, Bim/BCL2L11, } \\
\text { p21, Rbl2/p130 }\end{array}$ \\
\hline & Suppresseur de tumeurs & Cancer du sein [28] & $\mathrm{AlBl}$ \\
\hline Let-7 & Suppresseur de tumeurs & $\begin{array}{l}\text { Cancer du sein }[40] \text {, cancer des poumons }[29,31] \text {, cancers } \\
\text { hématopoïétiques [36] }\end{array}$ & $\begin{array}{l}\text { RAS, CDK6, CDC25A, } \\
\text { HMGA2, } \\
\text { C-MYC }\end{array}$ \\
\hline
\end{tabular}

Tableau I. miARN oncogéniques ou suppresseurs de tumeurs et types de cancer où on les trouve altérés. *Soutenus par des résultats expérimentaux.

Ainsi, plusieurs données chez la souris convergent pour indiquer un rôle pro-prolifératif et/ou pro-survie du polycistron miR-17-92.

Pour comprendre le rôle d'un miARN dans un processus cellulaire, il est nécessaire d'identifier également ses ARNm cibles. Les ARNm dont l'inhibition par le polycistron miR-17-92 a été vérifiée expérimentalement ont permis de mieux caractériser son rôle cellulaire et son potentiel oncogénique. II ressort que plusieurs des ARNm régulés par les miARN du polycistron miR17-92 codent pour des facteurs pro-apoptotiques ou des inhibiteurs du cycle cellulaire. Une des premières cibles identifiée du polycistron miR-17-92 est E2Fl $[17,18]$. II s'agit d'un facteur de transcription qui fait partie d'une famille de régulateurs du cycle cellulaire qui favorise la transition de la phase Gl à la phase $S$. De plus, le proto-oncogène $c-M y c$, lui aussi engagé dans la transition Gl-S, est directement responsable de l'induction de l'expression du polycistron miR-17-92, ce qui place ce polycistron au sein d'une des principales voies oncogéniques [17]. Deux études ont aussi montré que les facteurs de transcription $\varepsilon 2 F 1$ et $\varepsilon 2 F 3$ stimulent la transcription de miR-17-92 [18, 19]. Comme E2Fl et $\varepsilon 2 F 3$ sont des cibles du polycistron miR-17-92, cela suggère l'existence d'une boucle d'autorégulation entre ces facteurs de transcription et miR-17-92 (Figure 2). Compte tenu du fait qu'un haut niveau d'expression

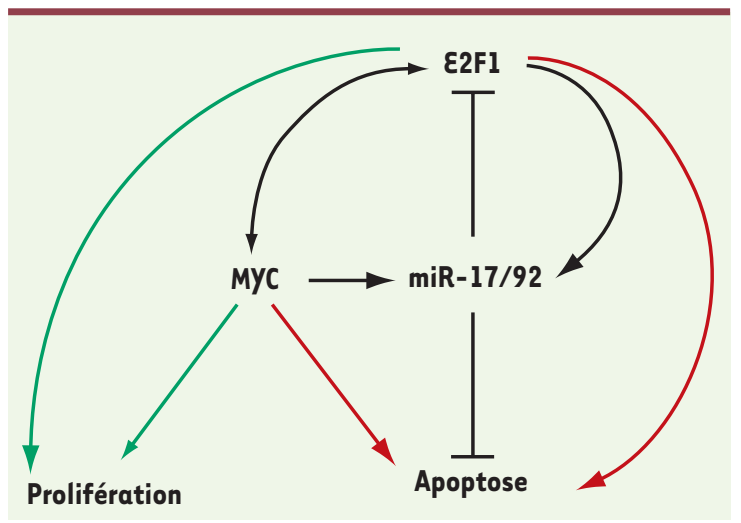

de c-Myc et $\varepsilon 2 F l$ peut entraîner l'apoptose, il est essentiel pour les cellules d'ajuster leurs niveaux d'expression. Le polycistron miR-17-92 pourrait restreindre l'activité $\varepsilon 2 F 1$ à un degré favorisant la prolifération cellulaire plutôt que l'apoptose.

D'autres ARNm cibles permettent d'expliquer la capacité oncogénique du polycistron. En effet, certains de ses ARNm cibles codent pour des inhibiteurs du cycle cellulaire et des suppresseurs de tumeurs, ce qui constituerait un mécanisme pro-prolifératif supplémentaire de ce polycistron. Ainsi, le facteur $\operatorname{CDKN1A/p21,~un~inhibiteur~de~l'activité~}$ kinase dépendante des cyclines et un régulateur négatif de la transition Gl-S, est aussi une cible de miR-17-92 [20], de même que $\mathrm{Rb} / 2 / \mathrm{p} 130$, un répresseur des facteurs de transcription $\varepsilon 2 \mathrm{~F}$, membre de la famille Rb et suppresseur de tumeurs [21]. Finalement, le suppresseur de tumeurs PTEN qui agit comme inhibiteur de la voie $\mathrm{PI}_{3}$ kinase et d'Akt/PKB est également régulé par le polycistron miR-17-92 [13]. Ce facteur complexe, qui est fréquemment muté dans plusieurs cancers [22], représente une cible des miARN d'intérêt majeur puisqu'il participe à une cascade de signalisation cellulaire limitant la prolifération cellulaire ou déclenchant l'apoptose.

Plusieurs études suggèrent que miR-17-92 exercerait un rôle dans le déclenchement de l'apoptose. Effectivement, l'inhibition des miARN miR-17-5 et miR-20a, issus du polycistron miR-17-92, provoque l'apoptose dans des cellules de cancer du poumon [23]. Le rôle de miR-17-92 dans la régulation de l'apoptose a été renforcé récemment par l'identification du facteur pro-apoptotique Bim comme cible de ce polycistron [12, 13, 24]. Bim régule la mort cellulaire en agissant comme antagoniste du facteur anti-apoptotique Bcl2. II est

Figure 2. Boucle autorégulatrice entre le polycistron miR-17-92 et les facteurs de transcription c-Myc et $\varepsilon 2 F 1$. Les protéines $\varepsilon 2 F 1$ et c-Myc s'activent mutuellement (flèche noire bidirectionnelle) et provoquent la transcription de miR17-92 (flèches noires). Cette activation permet, en retour, une régulation de l'expression de ces facteurs par le polycistron (traits noirs). Comme E2Fl et c-Myc peuvent promouvoir la prolifération cellulaire (flèches vertes) ou l'apoptose (flèches rouges) selon leur niveau d'expression, un modèle serait que miR-17-92 agirait comme un senseur du niveau d'activité de ces facteurs de transcription. Ainsi, en régulant l'expression de facteurs pro-apoptotiques ( $22 F 1, P T E N$ ) et anti-prolifératifs (p21), miR-17-92 favoriserait la prolifération cellulaire plutôt que l'apoptose dans des conditions normales de croissance cellulaire. 
particulièrement important lors de la maturation des lymphocytes $B$ puisqu'il participe à l'élimination des lymphocytes B autoréactifs [25]. Fait intéressant, Bim est connu pour agir comme suppresseur des lymphomes de cellules B induits par c-Myc; d'ailleurs, une des deux copies du gène Bim est fréquemment mutée dans ces lymphomes [26]. Ces résultats suggèrent que l'inhibition de Bim par les miARN du polycistron miR-17-92 pourrait expliquer en partie l'augmentation des lymphomes de type B chez les souris surexprimant à la fois c-Myc et ce polycistron. Cependant, comme nous l'avons décrit précédemment, le rôle anti-apoptotique du polycistron miR-17-92 ne constitue probablement pas la seule explication à la fonction pro-oncogénique de ces miARN.

Finalement, d'autres observations suggèrent de façon surprenante que miR-17-92 aurait aussi des propriétés anti-oncogéniques dans certains tissus. Le locus $13 q 31.3$, où est situé ce polycistron, présente fréquemment une délétion dans des cancers des ovaires, du sein et dans des mélanomes [27]. L'expression de miR-17-5, un miARN du polycistron miR-17-92, dans des lignées de cancer du sein, diminue leur prolifération [28]. Une cible de miR-17-5 pourrait être AIB-1, un co-activateur du récepteur des œstrogènes et de $\varepsilon 2 F 1$, essentiel à la croissance de certaines tumeurs du sein [28]. Ainsi, selon le contexte cellulaire et les ARNm cibles exprimés dans ces tissus, miR-17-92 aurait des propriétés oncogéniques ou de suppression de tumeurs. Cependant, il n'y a actuellement aucune preuve expérimentale montrant que miR-17-92 agit comme suppresseur de tumeurs.

\section{Let-7 : un miARN suppresseur de tumeurs}

Plusieurs miARN ont également été identifiés comme appartenant à la catégorie des suppresseurs de tumeurs et agissent, généralement, en réduisant l'expression d'oncogènes. Dans le cas des miARN, il semble qu'une réduction de leur expression atteignant un seuil critique soit suffisante pour engendrer une augmentation de l'oncogène ciblé et ainsi un phénotype de cancer. Let-7 illustre bien cette catégorie dans la mesure où il a été identifié comme inhibiteur du proto-oncogène RAS [29]. Chez l'humain, onze homologues du miARN let-7 sont connus et sont exprimés à partir de huit gènes différents, qui présentent fréquemment des délétions dans plusieurs cancers (Tableau I) [8]. L'analyse de ce miARN dans différents tissus a révélé que son expression était très forte dans les cellules de poumons sains [30]. En revanche, son niveau est fréquemment réduit dans des cancers du poumon,

Figure 3. c-Myc régule l'expression de plusieurs miARN impliqués dans des cancers. Les données récentes montrent que c-Myc induit la transcription de miARN oncogéniques, comme miR-17-92 (flèche noire) et réprime l'expression de miARN suppresseurs de tumeurs, comme let-7, miR-34 ou miR-15/16 (traits noirs) $[17,37]$. Plusieurs cancers expriment des niveaux très élevés de $c-M y c$, ce qui favoriserait la surexpression de miARN pro-oncogéniques et diminuerait l'expression de miARN suppresseurs de tumeurs. Cependant, le fait que les gènes de miARN oncogéniques comme miR-17-92 soient souvent amplifiés dans des tumeurs surexprimant $c-M y c$ suggère que cette régulation n'est pas suffisante en elle-même pour promouvoir la tumorigenèse. et celui-ci est concomitant à une surexpression de l'oncogène RAS, suggérant qu'il exercerait un rôle de suppresseur de tumeurs [29]. Cette observation permet ainsi de proposer un mécanisme pour l'action de let-7 dans les cancers. De plus, l'expression artificielle de ce miARN dans ce type de tumeurs entraîne une diminution de la prolifération des cellules [31]. D'autres ARNm ciblés par let-7 confirment l'importance de ce miARN pour la régulation de la croissance cellulaire. Par exemple, des facteurs essentiels pour la régulation du cycle cellulaire tels que les proto-oncogènes CDK6 et CDC25A sont des cibles validées de let-7 [32]. En diminuant leur expression, let-7 contrôlerait la transition de la phase Gl à S. Un autre oncogène caractérisé comme étant une cible de let-7 est HMGA2, protéine participant à la régulation de la transcription lors de la prolifération et de la différenciation cellulaires [33]. L'expression de HMGA2 est anormalement élevée dans plusieurs types de carcinomes, notamment dans les cancers des poumons où let- 7 est fréquemment inhibé. L'expression ectopique de let-7 a pour conséquence de réduire la synthèse du facteur HMGA2 et d'affecter la prolifération des cellules $[34,35]$. Enfin, let-7 régule également l'oncogène $\mathrm{c}-\mathrm{Myc}$, comme cela a été démontré dans des cancers hématopoïétiques du type lymphome de Burkitt [36]. L'expression du précurseur de let-7a diminue la synthèse de $c-M y c$ et ainsi l'expression des gènes régulés par Myc. Parmi les gènes réprimés par c-Myc, il est intéressant de noter que plusieurs codent pour let-7 [37], ce qui suggère que $c-M y c$ constitue un centre de régulation clé de l'expression de plusieurs micro-ARN dans la tumorigenèse (Figure 3 ).

\section{Discussion et conclusion}

Ces observations montrent que l'étude de la fonction d'un miARN est une tâche complexe. Dans la mesure où un même miARN peut cibler plusieurs ARNm, une variation dans le niveau d'un miARN peut avoir de larges répercussions sur l'expression de plusieurs gènes. Pour

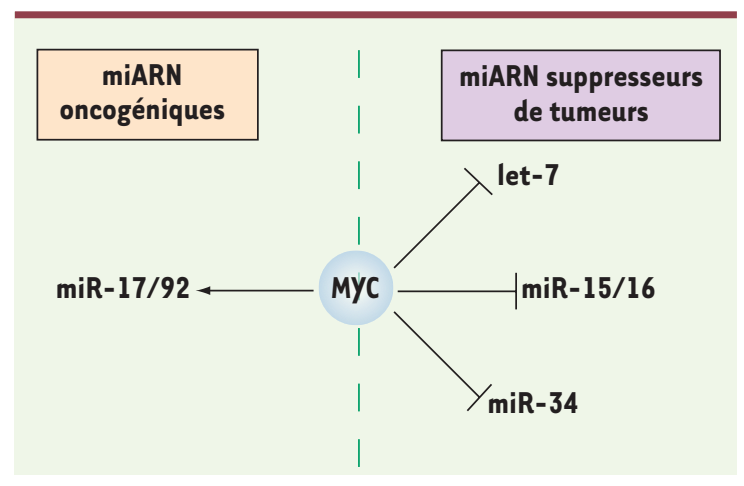


cette raison, les études futures chercheront à déterminer si ce sont seulement quelques ARNm cibles d'un miARN qui sont responsables de l'effet physiologique observé ou si c'est plutôt l'ensemble des ARNm cibles. Par exemple, une étude sur des souris transgéniques montre que la surexpression de let- 7 réduit la croissance de tumeurs chez ces souris [38]. L'expression ectopique de versions de RAS ou de HMGA2 sans leur 3'UTR ne restaure que partiellement la croissance tumorale chez ces souris, suggérant que des cibles autres que RAS et HMGA2 soient aussi responsables de l'action antitumorale de let-7. Dans le cas d'un polycistron de miARN, cette recherche se complexifie dans la mesure où plusieurs miARN peuvent coopérer entre eux pour réguler plusieurs $A R N m$. Les études du polycistron miR-17-92 réalisées dans différents types de tumeurs ont cependant permis d'apporter des éléments de réponse quant aux mécanismes d'action de tels polycistrons. Par exemple, miR-17-5 et miR-19, deux miARN de ce polycistron, coopéreraient pour réguler l'expression de PTEN [13]. Une autre complication vient aussi du fait qu'il existe plusieurs miARN redondants, comme les 11 homologues de let- 7 dans le génome humain. Sontils spécialisés, avec certains homologues de let-7 qui ne s'expriment que dans certains tissus, ou ont-ils des fonctions redondantes? De même, le polycistron miR17-92 a deux homologues dans les génomes humain et murin, miR-106a-363 et miR-106b-25. Alors que la délétion de miR-17-92 est létale chez la souris, la délétion de miR-106a-363 ou de miR-106b-25 produit des souris viables, suggérant des fonctions différentes entre ces polycistrons [12]. Finalement, le tissu dans lequel est exprimé un miARN affecterait aussi le type d'ARNm qu'il cible et le rôle de ce miARN. Comme cela est mentionné plus haut, alors que le polycistron miR-17-92 agit comme oncogène lorsqu'il est surexprimé dans certains cancers (lymphomes, cancers du poumon), il semble avoir des caractéristiques de suppresseur de tumeurs dans d'autres cancers (sein), où il serait plus sujet à délétions. Les études futures devront approfondir ces différents aspects pour mieux comprendre le rôle des miARN dans les cancers. $\diamond$

\section{SUMMARY}

Oncogenic and tumour suppressor microRNAs microRNAs constitute one of the most important discovery in the past few years in the field of gene expression regulation. They can precisely regulate the expression of a specific protein by inhibiting its translation and/ or promoting the degradation of its mRNA. In several cancers, the expression of some microRNAs is misregulated, pointing toward the existence of microRNAs with oncogenic or tumour suppressor properties. The miR-17-92 miRNA cluster has been reported to have a pro-oncogenic role in a mouse model system of Myc-induced B cell lymphoma. Some of its targets mRNAs code for proteins with pro-apoptotic or anti-proliferative functions, which shed some light on the mechanism of action of this cluster. On the other hand, a tumour suppressor miRNA like let-7 targets mRNAs coding for oncogenes and is frequently down-regulated in cancers. The finding that $\mathrm{c}-$ Myc controls the expression of several of these microRNAs reveals new information on how misregulation of this proto-oncogene can promote tumorigenesis. $\diamond$

\section{RÉFÉRENCES}

1. Lee RC, Feinbaum RL, Ambros V. The C. elegans heterochronic gene lin- 4 encodes small RNAs with antisense complementarity to lin-14. Cell $1993 ; 75: 843-54$.

2. Lewis BP, Burge CB, Bartel DP. Conserved seed pairing, often flanked by adenosines, indicates that thousands of human genes are microRNA targets. Cell $2005 ; 120: 15-20$.

3. Bartel DP. MicroRNAs: genomics, biogenesis, mechanism, and function. Cell 2004 ; $116: 281-97$.

4. Eulalio A, Huntzinger $\varepsilon$, Izaurralde $\varepsilon$. Getting to the root of miRNA-mediated gene silencing. Cell $2008 ; 132: 9-14$.

5. Vasudevan S, Tong $Y$, Steitz JA. Switching from repression to activation: microRNAs can upregulate translation. Science $2007 ; 318$ : 1931-4.

6. Ørom UA, Nielsen FC, Lund AH. MicroRNA-10a binds the 5'UTR of ribosomal protein mRNAs and enhances their translation. Mol Cell $2008 ; 30: 460-71$.

7. Calin GA, Croce CM. MicroRNA signatures in human cancers. Nat Rev Cancer $2006 ; 6: 857-66$.

8. Calin GA, Sevignani C, Dumitru CD, et al. Human microRNA genes are frequently located at fragile sites and genomic regions involved in cancers. Proc Natl Acad Sci USA 2004 ; $101: 2999-3004$.

9. He L, Thomson JM, Hemann MT, et al. A microRNA polycistron as a potential human oncogene. Nature $2005 ; 435: 828-33$.

10. Tanzer A, Stadler PF. Molecular evolution of a microRNA cluster. J Mol Biol 2004 ; 339 : 327-35

11. Mendell JT. miRiad roles for the miR-17-92 cluster in development and disease. Cell 2008 ; $133: 217-22$.

12. Ventura A, Young AG, Winslow MM, et al. Targeted deletion reveals essential and overlapping functions of the miR-17 92 family of miRNA clusters. Cell 2008; 132: 875-86.

13. Xiao C, Srinivasan L, Calado DP, et al. Lymphoproliferative disease and autoimmunity in mice with increased miR-17-92 expression in lymphocytes. Nat Immunol 2008 ; $9: 405-14$.

14. Hayashita $Y$, Osada H, Tatematsu $Y$, et al. A polycistronic microRNA cluster, miR-17-92, is overexpressed in human lung cancers and enhances cell proliferation. Cancer Res 2005 ; $65: 9628-32$.

15. Ota A, Tagawa H, Karnan S, et al. Identification and characterization of a novel gene, C13orf25, as a target for 13q31-q32 amplification in malignant lymphoma. Cancer Res 2004 ; $64: 3087-95$

16. Lu Y, Thomson JM, Wong HYF, et al. Transgenic over-expression of the microRNA miR-17-92 cluster promotes proliferation and inhibits differentiation of lung epithelial progenitor cells. Dev Biol 2007 ; 310 : 442-53.

17. O’Donnell KA, Wentzel $\varepsilon A$, Zeller KI, et al. c-Myc-regulated microRNAs modulate $\varepsilon 2 \mathrm{Fl}$ expression. Nature $2005 ; 435: 839-43$.

18. Sylvestre $Y$, De Guire V, Querido $\varepsilon$, et al. An $\varepsilon 2 F / m i R-20$ a autoregulatory feedback loop. J Biol Chem $2007 ; 282: 2135-43$.

19. Woods K, Thomson JM, Hammond SM. Direct regulation of an oncogenic micro-RNA cluster by E2F transcription factors. J Biol Chem $2007 ; 282$ : 2130-4.

20. Petrocca F, Visone R, Onelli MR, et al. E2Fl-regulated microRNAs impair TGFbeta-dependent cell-cycle arrest and apoptosis in gastric cancer. Cancer Cell $2008 ; 13: 272-86$.

21. Wang , Li YC, Wang J, et al. miR-17-92 cluster accelerates adipocyte differentiation by negatively regulating tumor-suppressor Rb2/p130. Proc Natl Acad Sci USA 2008 ; $105: 2889-94$.

22. Li J, Yen C, Liaw D, et al. PTEN, a putative protein tyrosine phosphatase gene mutated in human brain, breast, and prostate cancer. Science 1997 ; 275 : 1943-7.

23. Matsubara $H$, Takeuchi $T$, Nishikawa $\varepsilon$, et al. Apoptosis induction by antisense oligonucleotides against miR-17-5p and miR-20a in lung cancers overexpressing miR-17-92. Oncogene $2007 ; 26: 6099-105$.

24. Koralov SB, Muljo SA, Galler GR, et al. Dicer ablation affects antibody diversity and cell survival in the B lymphocyte lineage. Cell $2008 ; 132: 860-74$.

25. Bouillet $P$, Metcalf D, Huang DC, et al. Proapoptotic $B c l-2$ relative Bim required for certain apoptotic responses, leukocyte homeostasis, and to preclude autoimmunity. Science 1999 ; $286: 1735-8$. 
26. Egle A, Harris AW, Bouillet $P$, Cory $S$. Bim is a suppressor of Myc-induced mouse B cell leukemia. Proc Natl Acad Sci USA 2004 ; 101 : 6164-9.

27. Zhang L, Huang J, Yang $\mathrm{N}$, et al. microRNAs exhibit high frequency genomic alterations in human cancer. Proc Natl Acad Sci USA 2006 ; 103 : 9136-41.

28. Hossain A, Kuo MT, Saunders GF. Mir-17-5p regulates breast cancer cell proliferation by inhibiting translation of AlB1 mRNA. Mol Cell Biol $2006 ; 26: 8191-201$.

29. Johnson SM, Grosshans H, Shingara J, et al. RAS is regulated by the let-7 microRNA family. Cell $2005 ; 120: 635-47$.

30. Pasquinelli AE, Reinhart BJ, Slack F, et al. Conservation of the sequence and temporal expression of let-7 heterochronic regulatory RNA. Nature $2000 ; 408: 86-9$.

31. Takamizawa J, Konishi H, Yanagisawa K, et al. Reduced expression of the let-7 microRNAs in human lung cancers in association with shortened postoperative survival. Cancer Res 2004 ; $64: 3753-6$.

32. Johnson CD, Esquela-Kerscher A, Stefani G, et al. The let-7 microRNA represses cell proliferation pathways in human cells. Cancer Res 2007 ; $67: 7713-22$

33. Sgarra R, Rustighi A, Tessari MA, et al. Nuclear phosphoproteins HMGA and their relationship with chromatin structure and cancer. FEBS Lett $2004 ; 574: 1-8$.

34. Lee YS, Dutta A. The tumor suppressor microRNA let- 7 represses the HMGA2 oncogene. Genes Dev $2007 ; 21: 1025-30$.
35. Mayr C, Hemann MT, Bartel DP. Disrupting the pairing between let-7 and Hmga2 enhances oncogenic transformation. Science 2007 ; 315 : 1576-9.

36. Sampson VB, Rong NH, Han J, et al. MicroRNA Let-7a down-regulates MYC and reverts MYC-induced growth in Burkitt lymphoma Cells. Cancer Res $2007 ; 67: 9762-70$.

37. Chang TC, Yu D, Lee YS, et al. Widespread microRNA repression by Myc contributes to tumorigenesis. Nat Genet $2008 ; 40: 43-50$.

38. Kumar MS, Erkeland SJ, Pester RE, et al. Suppression of non-small cell lung tumor development by the let-7 microRNA family. Proc Natl Acad Sci USA $2008 ; 105: 3903-8$.

39. Volinia S, Calin GA, Liu CG, et al. A microRNA expression signature of human solid tumors defines cancer gene targets. Proc Natl Acad Sci USA $2006 ; 103: 2257-61$.

40. Yu F, Yao $\mathrm{H}$, Zhu $\mathrm{P}$, et al. let- 7 regulates self renewal and tumorigenicity of breast cancer cells. Cell $2007 ; 131: 1109-23$.

\section{TIRÉS À PART}

A.L. Finoux

\section{Collection SCIENCE ET BIOMÉDECINE}

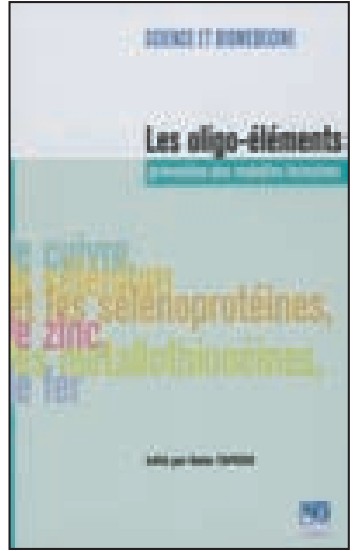

ISBN : 2-84254-107-3 64 pages

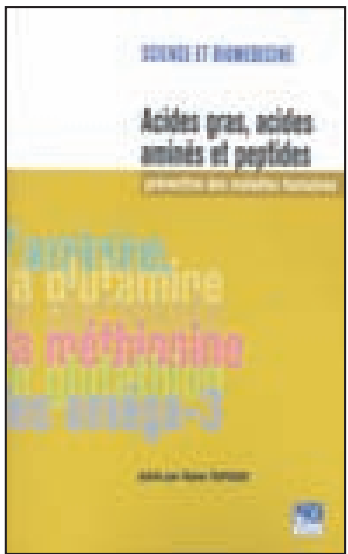

ISBN : 2-84254-108-1 80 pages

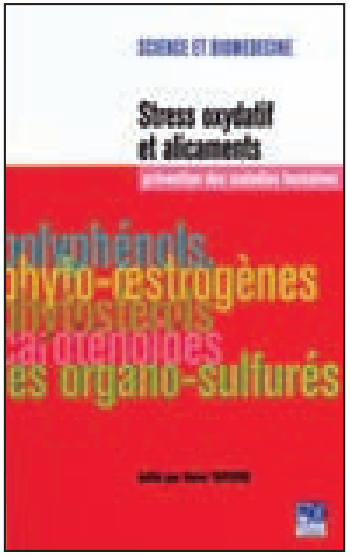

ISBN : 2-84254-111-1 86 pages

\section{Bon de commande}

À retourner à EDK, 2, rue Troyon - 92316 Sèvres Cedex

Tél. : 0155641393 - Fax : 0155641394 - E-mail : edk@edk.fr

NOM :

Prénom :.

Adresse :

Code postal :

Ville :

Pays :

Fonction :

Je souhaite recevoir l'ouvrage Les oligo-éléments : $10 €+3 €$ de port $=\mathbf{1 3} €$ TTC

Je souhaite recevoir l'ouvrage Acides gras, acides aminés et peptides : $12 €+3 €$ de port $=\mathbf{1 5} €$ TTC

Je souhaite recevoir l'ouvrage Stress oxydatif et alicaments : $14 €+3 €$ de port $=17 €$ TTC

en . exemplaire, soit un total de

$\square$ Par chèque, à l'ordre de $\mathbf{E} \mathbf{D}$ K

Par carte bancaire :

$\square$ Visa $\square$ Eurocard/Mastercard

Carte $n^{\circ} 1 \quad 1 \quad 1 \quad 1 \quad 1 \quad 1 \quad 1 \quad 1 \quad 1 \quad 1 \quad 1 \quad 1 \quad 1 \quad 1 \quad 1 \quad 1 \quad 1 \quad 1 \quad 1 \quad$

Date d'expiration: $\quad \underline{1 \quad 1 \quad 1 \quad 1}$

Signature :

$\mathrm{N}^{\circ}$ de contrôle au dos de la carte : 